\title{
Hard Corrections in Precision QCD for LHC and FCC Physics: A New Approach
}

\author{
B.F.L. Ward* \\ Baylor University \\ E-mail: bfl_wardebaylor.edu
}

\begin{abstract}
With an eye toward the usual unphysical divergence of hard fixed-order corrections in predictions for the processes probed in high energy colliding hadron beam devices as one approaches the soft limit, we present a new approach to the realization of such corrections, with some emphasis on the LHC and the future FCC devices. We show that the respective divergence is removed in our approach. This means that we would render the standard results to be closer to the observed exclusive distributions. While we stress that the approach has general applicability, we use the single $Z / \gamma$ production and decay to lepton pairs as our prototypical example. Accordingly, our work opens another part of the way to rigorous baselines for the determination of the theoretical precision tags for LHC physics, with an attendant generalization to the future FCC.
\end{abstract}

38th International Conference on High Energy Physics

3-10 August 2016

Chicago, USA

${ }^{*}$ Speaker. 


\section{Introduction}

In this current era of precision QCD, i.e., precision tags of $1 \%$ or better on QCD processes, we need rigorous baselines for the attendant theoretical predictions.In our exact amplitude resummation approach $[1,2,3,4]$ to such predictions, this amounts the construction of a semi-analytical realization of the predictions of the MC event generator used to make the predictions. This in turn requires an attendant realization of the corresponding hard gluon residuals in Refs. [1, 2, 3, 4] which derive from exact fixed order perturbation theory. Unfortunately, even though we have the exact NNLO result[5] for example for the single $Z / \gamma^{*}$ production and decay to lepton pairs, the soft limit of the calculation is at considerable variance with the data, as one can see in Refs. [6, 7]. It is clear that to address the precision of such results as those in [5], we need to tame the respective soft limits therein.

We can already see the problem at the NLO level. In order to properly assess the theoretical precision tag implied by our comparisons of MC@NLO[8]/Herwiri1.031[3] and MC@NLO/ Herwig6.5[9] vs the LHC data in Refs. [2, 3, 10], we need a baseline on the corresponding exact NLO results. The NLO version of the type of behavior we discussed for NNLO exact results is illustrated in Fig. 3 in Ref. [11] and is discussed in considerable detail in Ref. [12], where in eq.(5.5.30) of the latter reference it is shown that

$$
\frac{G_{p \rightarrow q}^{D Y}\left(x, Q^{2}\right)}{G_{p \rightarrow q}\left(x, Q^{2}\right)} \underset{x \rightarrow 1}{\rightarrow} 1+\frac{2 \alpha_{s}\left(Q^{2}\right)}{3 \pi} \ln ^{2}(1-x) .
$$

Here, $G_{p \rightarrow q}^{D Y}\left(G_{p \rightarrow q}\right)$ is the attendant Drell-Yan (DIS) structure function in the notation of Ref. [12]. This behavior in (1.1) is outside the scope of observable data, at the LHC or the newly conceived FCC, and calls into question what a precision tag could even mean?

In what follows, we present a new approach to hard corrections in QCD which will be seen to tame the divergent behavior in (1.1). The discussion will proceed as follows. In the next section, we give a brief review of the exact amplitude-based resummation theory that we use. In Section 3, we use the exact NLO Drell-Yan formula from Refs' $[13,14]$ to compute the corresponding exact amplitude-based resummed baseline NLO formula. Section 3 also contains our summary remarks. A longer version of the material presented here has already appeared in Ref. [11].

\section{Brief Review of Exact Amplitude-Based Resummation Theory}

The master formula for the exact amplitude-based resummation theory that we use is

$$
\begin{gathered}
d \bar{\sigma}_{\mathrm{res}}=e^{\mathrm{SUM}_{\mathrm{IR}}(\mathrm{QCED})} \sum_{n, m=0}^{\infty} \frac{1}{n ! m !} \int \prod_{j_{1}=1}^{n} \frac{d^{3} k_{j_{1}}}{k_{j_{1}}} \\
\prod_{j_{2}=1}^{m} \frac{d^{3} k^{\prime} j_{2}}{k^{\prime} j_{2}} \int \frac{d^{4} y}{(2 \pi)^{4}} e^{i y \cdot\left(p_{1}+q_{1}-p_{2}-q_{2}-\sum k_{j_{1}}-\sum k^{\prime} j_{2}\right)+D_{\mathrm{QCED}}} \\
\tilde{\bar{\beta}}_{n, m}\left(k_{1}, \ldots, k_{n} ; k_{1}^{\prime}, \ldots, k_{m}^{\prime}\right) \frac{d^{3} p_{2}}{p_{2}^{0}} \frac{d^{3} q_{2}}{q_{2}^{0}} .
\end{gathered}
$$

Here, $d \bar{\sigma}_{\text {res }}$ is either the reduced cross section $d \hat{\sigma}_{\text {res }}$ or the differential rate associated to a DGLAPCS $[15,16]$ kernel involved in the evolution of PDF's and where the new (YFS-style $[17,18]$ ) nonAbelian residuals $\tilde{\bar{\beta}}_{n, m}\left(k_{1}, \ldots, k_{n} ; k_{1}^{\prime}, \ldots, k_{m}^{\prime}\right)$ have $n$ hard gluons and $m$ hard photons and we show the final state with two hard final partons with momenta $p_{2}, q_{2}$ specified for a generic $2 f$ final state 
for definiteness. The infrared functions $\operatorname{SUM}_{\mathrm{IR}}(\mathrm{QCED}), D_{\mathrm{QCED}}$ are defined in Refs. $[4,19,20]$ to which we refer the reader accordingly. We would note that, as shown in Ref. [4], the new residuals $\tilde{\bar{\beta}}_{m, n}$ allow rigorous shower/ME matching via their shower subtracted analogs: in (2.1) we make the replacements

$$
\tilde{\bar{\beta}}_{n, m} \rightarrow \hat{\overline{\tilde{\beta}}}_{n, m}
$$

where the $\hat{\overline{\hat{\beta}}}_{n, m}$ have had all effects in the showers associated to the attendant PDF's $\left\{F_{j}\right\}$ removed from them. In this connection, we have in mind the standard formula a hard LHC(FCC) scattering process:

$$
d \sigma=\sum_{i, j} \int d x_{1} d x_{2} F_{i}\left(x_{1}\right) F_{j}\left(x_{2}\right) d \hat{\sigma}_{\text {res }}\left(x_{1} x_{2} s\right),
$$

where $d \hat{\sigma}_{\text {res }}$ is given in (2.1) and thus is consistent $[1,2,3,4]$ with our achieving a total precision tag of $1 \%$ or better for the total theoretical precision of (2.3).

The relationship between the residuals $\hat{\overline{\tilde{\beta}}}_{n, m}$ and the exact NLO corrections in the MC@NLO is given in eqs.(7-8) in Ref. [11]. From this relationship it follows that any study of the precision of results derived from (2.1) and used in (2.3) necessarily involves the study of the precision of the corresponding NLO exact results. If we have the behavior in (1.1), one would have to question the meaning of such a study in relation to LHC and FCC data. In the next section, we address this issue.

\section{Baseline Exact Amplitude-Based Resummed NLO Drell-Yan Formula}

Focusing on the $\gamma^{*}$ part of $Z / \gamma^{*}$ with just one flavor of unit charge for reasons of pedagogy, we recall from Refs. $[13,14]$ the exact NLO differential cross section formula

$$
\begin{aligned}
\frac{d \sigma^{D Y}}{d Q^{2}}= & \frac{4 \pi \alpha^{2}}{9 s Q^{2}} \int_{0}^{1} \frac{d x_{1}}{x_{1}} \int_{0}^{1} \frac{d x_{2}}{x_{2}}\left\{[ q ^ { ( 1 ) } ( x _ { 1 } ) \overline { q } ^ { ( 2 ) } ( x _ { 2 } ) + ( 1 \leftrightarrow 2 ) ] \left[\delta\left(1-z_{12}\right)\right.\right. \\
& \left.+\alpha_{s}(t) \theta\left(1-z_{12}\right)\left(\frac{1}{2 \pi} P_{q q}\left(z_{12}\right)(2 t)+f_{q}^{D Y}\left(z_{12}\right)\right)\right] \\
& +\left[\left(q^{(1)}\left(x_{1}\right)+\bar{q}^{(1)}\left(x_{1}\right)\right) G^{(2)}\left(x_{2}\right)+(1 \leftrightarrow 2)\right] \\
& \left.\times\left[\alpha_{s}(t) \theta\left(1-z_{12}\right)\left(\frac{1}{2 \pi} P_{q G}\left(z_{12}\right) t+f_{G}^{D Y}\left(z_{12}\right)\right)\right]\right\}
\end{aligned}
$$

where $z_{12}=\tau /\left(x_{1} x_{2}\right), \tau=Q^{2} / s$ in the usual conventions [12, 13, 14], the labels 1 and 2 refer to the two respective incoming protons and we follow the generic notation of Refs. [12, 13] here. In (3.1), unimproved DGLAP-CS $[15,16]$ kernels are

$$
\begin{aligned}
& P_{q q}(z)=C_{F}\left[\frac{1+z^{2}}{(1-z)_{+}}+\frac{3}{2} \delta(1-z)\right], \\
& P_{q G}(z)=\frac{1}{2}\left(z^{2}+(1-z)^{2}\right),
\end{aligned}
$$

where we define $t=\ln \left(Q^{2} / \mu^{2}\right)$ following Refs.[13, 12] so that $\mu$ is the 't Hooft [21] unity of mass. The scheme dependent hard correction terms are given as follows [13, 14] if one uses massless 
quarks and gluons and dimensional regularization, for example:

$$
\begin{aligned}
\alpha_{s} f_{G}^{D Y}(z)= & \frac{\alpha_{s}}{2 \pi} \frac{1}{2}\left[\left(z^{2}+(1-z)^{2}\right) \ln \frac{(1-z)^{2}}{z}-\frac{3}{2} z^{2}+z+\frac{3}{2}+2 P_{q G}(z) \zeta\right] \\
\alpha_{s} f_{q}^{D Y}(z)= & C_{F} \frac{\alpha_{s}}{2 \pi}\left[4\left(1+z^{2}\right)\left(\frac{\ln (1-z)}{1-z}\right)_{+}-2 \frac{1+z^{2}}{1-z} \ln z\right. \\
& \left.+\left(\frac{2 \pi^{2}}{3}-8\right) \delta(1-z)+\frac{2}{C_{F}} P_{q q}(z) \zeta\right]
\end{aligned}
$$

where we define [14] $\zeta=-\frac{1}{\varepsilon}+C_{E}-\ln 4 \pi$ for $\varepsilon=2-n / 2$ when $n$ is the dimension of space-time. $C_{E}$ is Euler-Mascheroni constant. In the $\overline{\mathrm{MS}}$ scheme, the terms proportional to $\zeta$ are removed by mass factorization, which also replaces $\mu$ by $\Lambda$ in $t$ following Ref. [12]. This leaves the +-functions in the hard corrections and it is the divergent behavior of these distributions as $z \rightarrow 1$ that produces the attendant unphysical results referenced above. .

To fix this, we imbed the calculation of the hard correction terms into the master formula (2.1) restricted to its QCD aspect. This gives the following resummed version of (3.1):

$$
\begin{aligned}
\frac{d \sigma_{\text {res }}^{D Y}}{d Q^{2}}= & \frac{4 \pi \alpha^{2}}{9 s Q^{2}} \int_{0}^{1} \frac{d x_{1}}{x_{1}} \int_{0}^{1} \frac{d x_{2}}{x_{2}}\left\{\left[q^{(1)}\left(x_{1}\right) \bar{q}^{(2)}\left(x_{2}\right)+(1 \leftrightarrow 2)\right] 2 \gamma_{q} F_{Y F S}\left(2 \gamma_{q}\right)\left(1-z_{12}\right)^{2 \gamma_{q}-1} e^{\delta_{q}}\right. \\
& \times \theta\left(1-z_{12}\right)\left[1+\gamma_{q}-7 C_{F} \frac{\alpha_{s}}{2 \pi}+\left(1-z_{12}\right)\left(-1+\frac{1-z_{12}}{2}\right)\right. \\
& +2 \gamma_{q}\left(-\frac{1-z_{12}}{2}-\frac{z_{12}^{2}}{4} \ln z_{12}\right) \\
& \left.+\alpha_{s}(t) \frac{\left(1-z_{12}\right)}{2 \gamma_{q}} f_{q}^{D Y}\left(z_{12}\right)\right] \\
& +\left[\left(q^{(1)}\left(x_{1}\right)+\bar{q}^{(1)}\left(x_{1}\right)\right) G^{(2)}\left(x_{2}\right)+(1 \leftrightarrow 2)\right] \\
& \times \gamma_{G} F_{Y F S}\left(\gamma_{G}\right) e^{\frac{\delta_{G}}{2}}\left[\alpha _ { s } ( t ) \theta ( 1 - z _ { 1 2 } ) \left(\frac{t}{2 \pi \gamma_{G}}\left(\frac{1}{2}\left(z_{12}^{2}\left(1-z_{12}\right)^{\gamma_{G}}+\left(1-z_{12}\right)^{2} z_{12}^{\gamma_{G}}\right)\right)\right.\right. \\
& \left.\left.\left.+f_{G}^{D Y^{\prime}}\left(z_{12}\right) / \gamma_{G}\right)\right]\right\}
\end{aligned}
$$

where we have introduced here

$$
\begin{aligned}
\alpha_{s} f_{G}^{D Y^{\prime}}(z)= & \frac{\alpha_{s}}{2 \pi} \frac{1}{2}\left[\left(z^{2}(1-z)^{\gamma_{G}}+(1-z)^{2} z^{\gamma_{G}}\right) \ln \frac{(1-z)^{2}}{z}-\frac{3}{2} z^{2}(1-z)^{\gamma_{G}}+z(1-z)^{\gamma_{G}}\right. \\
& \left.+\frac{3}{4}\left((1-z)^{\gamma_{G}}+z^{\gamma_{G}}\right)\right]
\end{aligned}
$$

and the following exponents and YFS infrared function, $F_{\mathrm{YFS}}$, already needed for the IR-improvement of DGLAP-CS theory in Refs. [19, 20]:

$$
\begin{aligned}
\gamma_{q} & =C_{F} \frac{\alpha_{s}}{\pi} t=\frac{4 C_{F}}{\beta_{0}}, & \delta_{q} & =\frac{\gamma_{q}}{2}+\frac{\alpha_{s} C_{F}}{\pi}\left(\frac{\pi^{2}}{3}-\frac{1}{2}\right), \\
\gamma_{G} & =C_{G} \frac{\alpha_{s}}{\pi} t=\frac{4 C_{G}}{\beta_{0}}, & \delta_{G} & =\frac{\gamma_{G}}{2}+\frac{\alpha_{s} C_{G}}{\pi}\left(\frac{\pi^{2}}{3}-\frac{1}{2}\right), \\
F_{\mathrm{YFS}}(\gamma) & =\frac{e^{-C_{E} \gamma}}{\Gamma(1+\gamma)} . & &
\end{aligned}
$$


We define $\beta_{0}=11-\frac{2}{3} n_{f}$ for $n_{f}$ active flavors in a standard way and $\Gamma(w)$ is Euler's gamma function of the complex variable $w$. We have mass factorized in (3.4) and (3.5) as indicated above. We see that the regime at $z_{12} \rightarrow 1$ is now under control in (3.4) so that we will no longer have the unphysical behavior discussed above. This is the main result of this paper.

More precisiely, in lieu of the result in (1.1), we get the behavior such that the $\ln ^{2}(1-x)$ on the RHS of (1.1) is replaced by

$$
\frac{2(1-x)^{\gamma_{q}} \ln (1-x)}{\gamma_{q}}-\frac{2(1-x)^{\gamma_{q}}}{\gamma_{q}^{2}}
$$

and this vanishes for $x \rightarrow 1$. Our result means that the hard correction now has the possibility to be compared rigorously to the data in an exclusive manner. We take up such matters elsewhere. [22].

We note that the parton shower/ME matching formulas in MC@NLO and in POWHEG [23] do not remove the IR divergence which we just tamed - the latter retains the NLO correction with its bad IR limit in the soft regime for $z_{12} \rightarrow 1$ and the former replaces the bad IR behavior of the NLO correction with that of the unimproved parton shower real emission at the same order which is infrared divergent for $z_{12} \rightarrow 1$ and requires an ad hoc IR cut-off $k_{0}$-parameter, as we have discussed in Ref. [3]. No such parameter is needed in our new approach.

In summary, we have introduced a new approach to hard corrections in perturbative QCD which allows the realization of the same type of semi-analytical baselines for QCD that we and our collaborators had in Refs. [17] for the higher order corrections in the Standard Model EW theory. We look with excitement to its exploitation in precision LHC and FCC physics scenarios. In closing, we thank Prof. Ignatios Antoniadis for the support and kind hospitality of the CERN TH Unit while part of this work was completed.

\section{References}

[1] B.F.L. Ward et al., PoS(RADCOR2013)(2014) 054.

[2] S.K. Majhi et al., Ann. Phys. 350 (2014) 485.

[3] S. Joseph et al., Phys. Lett. B685 (2010) 283; Phys. Rev. D81 (2010) 076008; B.F.L. Ward et al., Mod. Phys. Lett. A25 (2010) 2207; B.F.L. Ward and S. Yost, PoS (ICHEP2010) (2011) 127; B.F.L. Ward, S.K. Majhi and S.A. Yost, PoS(RADCOR2011) (2012) 022; S.K. Majhi et al., Phys. Lett. B719 (2013) 367; and references therein.

[4] C. Glosser, S. Jadach, B.F.L. Ward and S.A. Yost, Mod. Phys. Lett. A19 (2004) 2113; B.F.L. Ward, C. Glosser, S. Jadach and S.A. Yost, in Proc. DPF 2004, Int. J. Mod. Phys. A 20 (2005) 3735; in Proc. ICHEP04, vol. 1, eds. H. Chen et al.,(World. Sci. Publ. Co., Singapore, 2005) p. 588; B.F.L. Ward and S. Yost, preprint BUHEPP-05-05, in Proc. HERA-LHC Workshop, CERN-2005-014; in Moscow 2006, ICHEP, vol. 1, p. 505; Acta Phys. Polon. B 38 (2007) 2395; arXiv:0802.0724,

PoS(RADCOR2007)(2007) 038; B.F.L. Ward et al., arXiv:0810.0723, in Proc. ICHEP08; arXiv:0808.3133, in Proc. 2008 HERA-LHC Workshop, DESY-PROC- 2009-02, eds. H. Jung and A. De Roeck, (DESY, Hamburg, 2009)pp. 180-186, and references therein.

[5] K. Melnikov and F. Petriello, Phys. Rev. D 74 (2006)114017; R. Gavin et al., arXiv:1101.3540; and references therein.

[6] S. Hassani, in Proc. Recntres de Moriond EW, 2013, in press; G. Aad et al., arXiv:1211.6899, and references therein. 
[7] H. Yin, in Proc. Recntres de Moriond EW, 2013, in press; T. Aaltonen et al., Phys. Rev. D 86 (2012) 052010, and references therein.

[8] S. Frixione and B.Webber, J. High Energy Phys. 0206 (2002) 029; S. Frixione et al., arXiv:1010.0568; B. Webber, talk at CERN, 03/30/2011; S. Frixione, talk at CERN, 05/04/11.

[9] G. Corcella et al., hep-ph/0210213; J. High Energy Phys. 0101 (2001) 010; G. Marchesini et al., Comput. Phys. Commun.67 (1992) 465.

[10] A. Mukhopadhyay and B.F.L. Ward, Mod. Phys. Lett. A 31 (2016) 1650063.

[11] B.F.L. Ward, Mod. Phys. Lett. A 31 (2016) 1650126.

[12] R.D. Field, Applications of Perturbative QCD,(Addison-Wesley Publ. Co., Inc, Redwood City, 1989).

[13] G. Altarelli, R.K. Ellis and G. Martinelli, Nucl.Phys. B 157 (1979) 461, and references therein.

[14] B. Humpert and W.L. Van Neerven, Nucl. Phys. B 184 (1981) 225, and references therein.

[15] G. Altarelli and G. Parisi, Nucl. Phys. B 126 (1977) 298; Yu. L. Dokshitzer, Sov. Phys. JETP 46 (1977) 641; L. N. Lipatov, Yad. Fiz. 20 (1974) 181; V. Gribov and L. Lipatov, Sov. J. Nucl. Phys. 15 (1972) 675, 938; see also J.C. Collins and J. Qiu, Phys. Rev. D 39 (1989) 1398.

[16] C.G. Callan, Jr., Phys. Rev. D 2 (1970) 1541; K. Symanzik, Commun. Math. Phys. 18 (1970) 227, and in Springer Tracts in Modern Physics, 57, ed. G. Hoehler (Springer, Berlin, 1971) p. 222; see also S. Weinberg, Phys. Rev. D 8 (1973) 3497.

[17] See also S. Jadach and B.F.L. Ward, Comput. Phys. Commun. 56(1990) 351; Phys.Lett. B 274 (1992) 470; S. Jadach et al., Comput. Phys. Commun. 102 (1997) 229; S. Jadach, W. Placzek and B.F.L Ward, Phys. Lett. B 390 (1997) 298; S. Jadach, M. Skrzypek and B.F.L. Ward, Phys. Rev. D 55 (1997) 1206; S. Jadach, W. Placzek and B.F.L. Ward, Phys. Rev. D 56 (1997) 6939; S. Jadach, B.F.L. Ward and Z. Was, Phys. Rev. D 63 (2001) 113009; Comp. Phys. Commun. 130 (2000) 260; ibid.124 (2000) 233; ibid.79 (1994) 503; ibid.66 (1991) 276; S. Jadach et al., ibid.140 (2001) 432, 475; see also S. Jadach, B.F.L. Ward and Z. Was, Phys. Rev. D88 (2013) 114022.

[18] D. R. Yennie, S. C. Frautschi, and H. Suura, Ann. Phys. 13 (1961) 379; see also K. T. Mahanthappa, Phys. Rev. 126 (1962) 329, for a related analysis.

[19] B.F.L. Ward, Adv. High Energy Phys. 2008 (2008) 682312.

[20] B.F.L. Ward, Ann. Phys. 323 (2008) 2147.

[21] G. âĂŹt Hooft, Nucl. Phys. B 61 (1973) 455, and references therein.

[22] B.F.L. Ward, to appear.

[23] S. Alioli et al., J. High Energy Phys. 1006 (2010) and references therein. 